DRAFT VERSION NOVEMBER 5, 2018

Preprint typeset using LTEX style emulateapj v. 04/20/08

\title{
PHOTOSPHERIC EMISSION AS THE DOMINANT RADIATION MECHANISM IN LONG-DURATION GAMMA-RAY BURSTS
}

\author{
Davide Lazzati $^{1}$, Brian J. Morsony ${ }^{2}$, Raffaella Margutti ${ }^{3}$, And Mitchell C. Begelman ${ }^{4,5}$ \\ Draft version November 5, 2018
}

\begin{abstract}
We present the results of a set of numerical simulations of long-duration gamma-ray burst jets associated with massive, compact stellar progenitors. The simulations extend to large radii and allow us to locate the region in which the peak frequency of the advected radiation is set before the radiation is released at the photosphere. Light curves and spectra are calculated for different viewing angles as well as different progenitor structures and jet properties. We find that the radiation released at the photosphere of matter-dominated jets is able to reproduce the observed Amati and energy-Lorentz factor correlations. Our simulations also predict a correlation between the burst energy and the radiative efficiency of the prompt phase, consistent with observations.
\end{abstract}

Subject headings: gamma-ray: bursts — hydrodynamics — methods: numerical — relativity

\section{INTRODUCTION}

Long-duration gamma-ray bursts (GRBs) are characterized by bright, non-thermal radiation spectra with typical photon energies of a few hundred $\mathrm{keV}$ to several MeV (Band et al 1993; Kaneko et al. 2006; Zhang et al. 2011). The standard model to explain these observations invokes collisionless shocks between parts of the relativistic outflows moving at different speed (Rees \& Meszaros 1994) to produce a tangled magnetic field and non-thermal electrons, eventually releasing synchrotron radiation (Daigne \& Mochkovitch 1998; Piran 1999, Bošnjak et al. 2009). Alternatively, the magnetic field may be advected from the central engine and the collisions working as a trigger for magnetic dissipation (the ICMART model, Zhang \& Yan 2011).

When GRB observations are corrected for redshift and considered in the burst's frame, a correlation between the burst's isotropic equivalent energy and its characteristic photon energy is revealed (Amati et al. 2002). The correlation was originally discovered with data from BeppoSAX, and dubbed the Amati correlation. Even though the role of selection effects has not been completely understood (Nakar \& Piran 2005; Kocevski 2012), the correlation has been confirmed with data from all subsequent observatories (Amati 2006; Ghirlanda et al. 2008; Amati et al. 2009). To lend credibility to an intrinsic origin, a correlation between burst peak luminosity and peak photon energy is also observed among bursts (Yonetoku et al. 2004; Ghirlanda et al. 2012a) and within individual events ( $\mathrm{Lu}$ et al. 2012). The latter correlation is free of selection effects and points to a physical origin for all the others. More recently, the analysis of a sample of GRBs with very early afterglow observations revealed a correlation between the isotropic equivalent energy of the prompt emission and the Lorentz factor of the outflow (Liang et al. 2010; Ghirlanda et al. 2012b). Taken together, these correlations present a

\footnotetext{
${ }^{1}$ Department of Physics, NC State University, 2401 Stinson Drive, Raleigh, NC 27695-8202

2 Department of Astronomy, University of Wisconsin-Madison, 3321 Sterling Hall, 475 N. Charter Street, Madison WI 53706-1582

${ }^{3}$ Harvard-Smithsonian Center for Astrophysics, ITC, 60 Garden Street, Cambridge, MA 02138, USA

${ }^{4}$ JILA, University of Colorado and National Institute of Standards and Technology, Boulder, CO 80309-0440, USA

${ }^{5}$ Department of Astrophysical and Planetary Sciences, University of Colorado, Boulder, CO 80309-0391, USA
}

challenge to the standard synchrotron shock model (SSM). Outflows characterized by large Lorentz factors should produce internal shocks at large radii, therefore generating magnetic fields of much lower intensity, and eventually bursts characterized by smaller photon energies, predicting an anticorrelation in contrast to observations (Ghisellini et al. 2000).

An alternative model for the prompt emission of GRBs is the photospheric model (Mészáros \& Rees 2000; Pe'er et al. 2005, 2006; Rees \& Mészáros 2005; Giannios 2006; Lazzati et al. 2009). It does not specify how the photons are produced, concentrating instead on how the interaction of the radiation field with the leptonic component of the outflow modifies the spectrum in the optically thick phase, before it is released at the photosphere. The photospheric model has been proposed as a viable candidate to reproduce correlations such as the Amati correlation on the qualitative level (Thompson et al. 2007; Lazzati et al. 2011; Fan et al. 2012). On the quantitative side, comparison of the burst properties from numerical simulations to observations have shown that while photospheric emission from baryonic jets can reproduce the slope of the correlation, they cannot reproduce the normalization, simulated bursts being more energetic than observed ones for a given peak frequency (Lazzati et al. 2011; Nagakura et al. 2011). Deeper theoretical understanding of photospheric emission has revealed that radiation and matter are not in equilibrium all the way to the photosphere and therefore the peak of the spectrum is formed at moderate optical depths $(\tau \sim 50)$ when the radiation is at higher temperature (Giannios 2012).

In this paper we present results of an extensive set of simulations aimed at exploring quantitatively the predictions of the photospheric model for baryonic jets. In contrast to our previous work, the peak frequency of the spectrum is calculated at an optical depth larger than unity, following Giannios (2012). We also explore the effect of the jet injection properties and of the progenitor structure on the ensuing light curves and spectra. This paper is organized as follows: in Sect. 2 we present our numerical simulations, in Sect. 3 we detail the post processing methods to derive light curves and spectra, in Sect. 4 we present our results, and in Sect. 5 we summarize and discuss this work.

\section{NUMERICAL SIMULATIONS}

All the simulations presented in this paper were performed with the FLASH code (Fryxell et al. 2000), version 2.5. The 


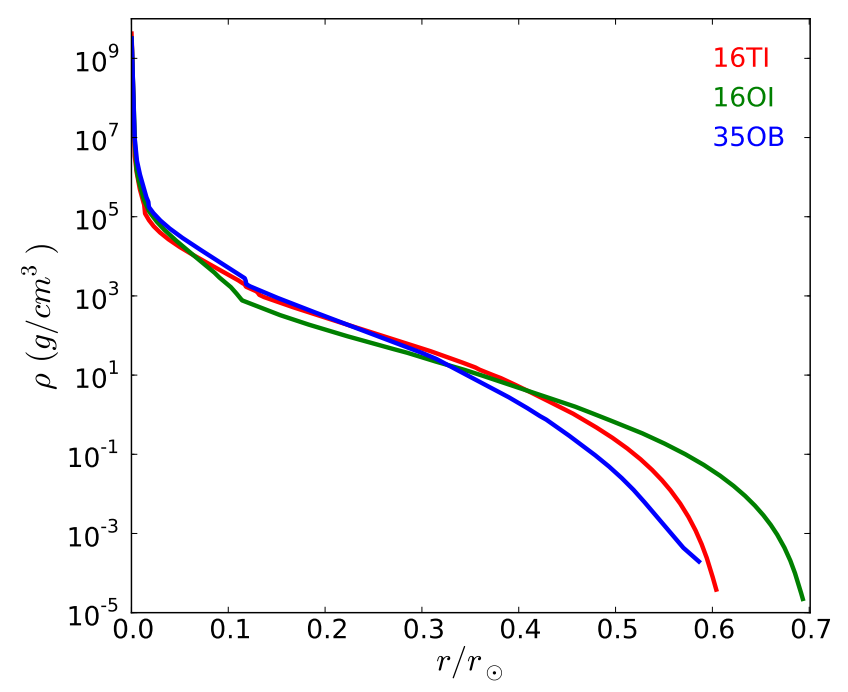

FIG. 1.- Density profiles of the GRB progenitor stars used in this work.

FLASH code is a modular block-structured adaptive mesh refinement code, parallelized using the Message Passing Interface (MPI) library. It solves the Riemann problem using the Piecewise Parabolic Method (PPM). From the point of view of the research presented here, the main strength of this code is that it can perform special relativistic hydrodynamic computations on an adaptive mesh. This is crucial for the modeling of the interaction of the collimated jet with the stellar envelope. This approach allows for significantly enhanced resolution along the jet axis and the jet-star boundary without the need to resolve the rest of the star to the same extent, thereby making high-resolution simulations feasible. In order to increase the resolution near the center of the star and close to the axis of the jet we modified FLASH to be able to vary the maximum level of refinement allowed over the simulation grid (see also López-Cámara et al. 2013). Additional modifications to the standard FLASH code are described in Morsony et al. (2007).

We adopted a maximum resolution of $4 \times 10^{6} \mathrm{~cm}$ at the highest level of refinement. At this resolution the transverse dimension of the injected jet is resolved into 44 elements. Our simulations do not include magnetic fields, due to the technical challenge of performing MHD calculations with relativistic motions on an adaptive mesh. In addition, gravity from a central mass and self-gravity are neglected since the characteristic times of the jet-star interaction are much shorter than the dynamical time of the progenitor star's collapse.

All our simulations adopted a realistic GRB stellar progenitor, and we explored three different progenitors all taken from Woosley \& Heger (2006). Model 16TI is a 16 solarmass Wolf-Rayet star with an initial metallicity $1 \%$ solar and angular momentum $J=3.3 \times 10^{52} \mathrm{erg} \cdot \mathrm{s}$. The mass of the star at pre-explosion is 13.95 solar masses and its radius is $4.07 \times 10^{10} \mathrm{~cm}$, corresponding to 0.6 solar radii. A relativistic jet is injected as a boundary condition at a distance $R_{0}=10^{9} \mathrm{~cm}$ from the star's center. The jet has initial Lorentz factor $\Gamma_{0}=5$ and an half-opening angle $\theta_{0}=10^{\circ}\left(5^{\circ}\right.$ in one case). The jet is injected hot, with enough internal energy to allow for acceleration up to an asymptotic Lorentz factor $\Gamma_{\infty}=400$ upon complete, non-dissipative acceleration ( $\Gamma_{\infty}=100$ upper limit was adopted in one case). The en-

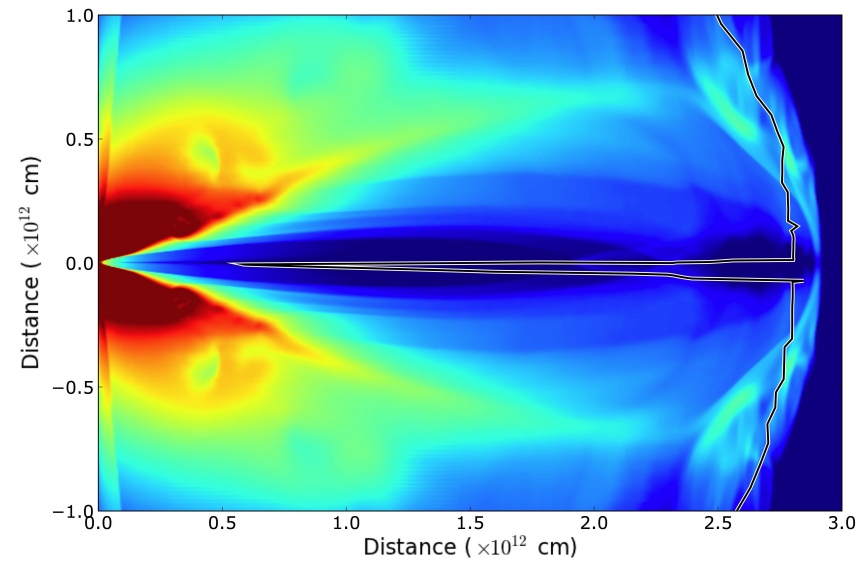

FIG. 2.- False-color stratification map of the logarithm of the density of our fiducial 16TI simulation at $t=100 \mathrm{~s}$. The thick black line shows the location of the photosphere for an observer located at a viewing angle $\theta_{o}=$ $0.5^{\circ}$ towards the bottom of the panel.

\begin{tabular}{c|c|c|c|c|c|c} 
Sim. \# & Prog. ID & $\Gamma_{0}$ & $\Gamma_{\infty}$ & $\theta_{0}$ & $L_{\text {jet }}(\mathrm{erg} / \mathrm{s})$ & $t_{\text {eng }}(\mathrm{s})$ \\
\hline 1 & $16 \mathrm{TI}$ & 5 & 400 & 10 & $5.33 \times 10^{50}$ & 100 \\
2 & $16 \mathrm{TI}$ & 5 & 100 & 10 & $10^{50}$ & 100 \\
3 & $35 \mathrm{OB}$ & 5 & 400 & 10 & $5.33 \times 10^{50}$ & 100 \\
4 & $16 \mathrm{OI}$ & 5 & 400 & 10 & $5.33 \times 10^{50}$ & 100 \\
5 & $16 \mathrm{TI}$ & 5 & 400 & 10 & $10^{50}$ & 100 \\
6 & $16 \mathrm{TI}$ & 5 & 400 & 5 & $5.33 \times 10^{50}$ & 100 \\
7 & $16 \mathrm{TI}$ & 5 & 400 & 10 & $5.33 \times 10^{50}$ & 67 \\
8 & $16 \mathrm{TI}$ & 5 & 400 & 10 & $5.33 \times 10^{50}$ & 30
\end{tabular}

TABLE 1

DETAILS OF THE SIMULATIONS PRESENTED IN THIS WORK.

gine luminosity is kept constant for a duration $t_{\text {eng }}$ (typically $t_{\text {eng }}=100 \mathrm{~s}$, but shorter engines were explored as well), after which the jet is turned off and the boundary condition is set to reflective. The bottom and polar boundaries are set to reflective for the whole duration of the simulation, while the two outer boundaries are set to absorbing. The simulation box is $2.5 \times 10^{13} \mathrm{~cm}$ in length (along the jet direction) and $5 \times 10^{12} \mathrm{~cm}$ across, approximately a factor 10 larger than our previous simulations.

Two additional progenitor stars were tested. Model $16 \mathrm{OI}$ is a star of 16 solar masses with an initial metallicity $10 \%$ solar. Its pre-explosion mass is 12.21 solar masses and its radius is 0.7 solar radii. Model $35 \mathrm{OB}$ is a 35 solar-mass star with initial metallicity $10 \%$ solar, pre-explosion mass 21.24 solar masses, and radius 0.6 solar radii. The density profiles of the three progenitors are shown in Figure 1, while details of the simulations are reported in Table 1 .

\section{SPECTRA AND LIGHT CURVES}

The calculation of the light curves and spectra is performed analogously to Lazzati et al. (2011, see also Mizuta et al. 2011; Nagakura et al. 2011) with a few important changes. First, we changed the optical depth at which we compute the radiation temperature. In our previous publications, we used the photospheric radius as the location at which the radiation temperature was calculated through the black body relation $u=a T^{4}$, where $u$ is the energy density and $a=7.56 \times 10^{-15} \mathrm{erg} \mathrm{cm}^{-3} \mathrm{~K}^{-4}$ is the radiation energy constant. We now use Eq. 6 from Giannios (2012) to derive the 
opacity at which the radiation temperature is set:

$$
\tau_{T}=46 \frac{L_{53}^{1 / 6} f_{ \pm}^{1 / 3}}{\Gamma_{2.5}^{1 / 3} \epsilon^{1 / 6} \eta_{2.5}^{1 / 3}}
$$

where $f_{ \pm}$is the number of leptons per proton (including electron-positron pairs), $\epsilon$ is the fraction of the energy of the outflow carried by radiation, $\eta=L / \dot{M} c^{2}$ is the asymptotic Lorentz factor, and we used the notation $Q_{x}=10^{-x} Q$. Because of the decrease in the radius with respect to the standard assumption $\tau=1$, the location at which the spectrum is formed is easier to determine from the simulation frames and no correction needs to be applied (see Eq. 4 in Lazzati et al. 2011). Our numerical simulations do not allow us to compute the number of electron-positron pairs and we assume that $f_{ \pm}=1$ for the remainder of this paper. This assumption is justified by the fact that the comoving temperature is of only a few $\mathrm{keV}$ or less where the peak frequency is computed and, in absence of non-thermal radiation, pairs are not important. If a significant population of pairs were present, the location of the spectrum formation would be moved outwards and the peak frequency would be decreased, weakening the agreement with observations (see figures and discussion below).

The location of the region of the spectral peak formation was calculated analogously to Lazzati et al. (2011) but updating their condition to:

$$
\begin{aligned}
& 46 \frac{L_{53}^{1 / 6} f_{ \pm}^{1 / 3}}{\Gamma_{2.5}^{1 / 3} \epsilon^{1 / 6} \eta_{2.5}^{1 / 3}}= \\
& =-\int_{Z_{\mathrm{obs}}}^{Z_{\mathrm{ph}}(x)} \sigma_{T} n^{\prime}\left(t_{\mathrm{obs}}-\frac{Z_{\mathrm{obs}}-z}{c}, x, z\right) \Gamma\left[1-\beta \cos \left(\theta_{v}\right)\right] d z(2)
\end{aligned}
$$

where $\beta \equiv \beta\left(t_{\mathrm{lab}}, x, z\right)$ is the local velocity of the outflow in units of the speed of light, $\Gamma \equiv \Gamma\left(t_{\text {lab }}, x, z\right)$ is the local bulk Lorentz factor, and $\theta_{v} \equiv \theta_{v}\left(t_{\mathrm{lab}}, x, z\right)$ is the angle between the velocity vector and the direction of the line of sight. $x$ is the coordinate perpendicular to the line of sight, while $z$ is the coordinate along the line of sight. All the values of $\beta, \Gamma$, and $\theta_{v}$ are evaluated at the same delayed coordinate $\left(t_{\mathrm{lab}}, x, z\right) \equiv$ $\left(t_{\mathrm{obs}}-\frac{Z_{\mathrm{obs}}-z}{c}, x, z\right)$ as the comoving density.

Figure 2 shows an image of the density stratification from our fiducial simulation (simulation number 1 in Table 1) at a time $t=100 \mathrm{~s}$ after the engine onset. A black line is used to show the location of the photosphere for an observer located at a viewing angle $\theta_{o}=0.5^{\circ}$ towards the bottom of the panel. Most of the photospheric emission comes from the bottom of the trough where the outflow velocity vector and the radiation propagation are aligned within an angle $\sim 1 / \Gamma$.

In addition, we refined the way in which we compute the peak frequency to make the comparison with observations more accurate. First, we consider the fact that what is measured from the data is the peak of the $\nu F(\nu)$ spectrum. Second, we consider the fact that the location of the peak frequency is affected by the addition of high-frequency powerlaw tails likely due to Comptonization (Pe'er et al. 2006; Giannios \& Spruit 2007; Beloborodov 2010; Lazzati \& Begelman 2010) and low-frequency power-laws likely due to a synchrotron component (Vurm et al. 2011). The combination of these two effects shifts the peak to higher frequencies by approximately a factor 2 , depending on the slope of the highand low- frequency power-law components (see Figure 3 ).

A sample of light curves, photospheric radii, and peak frequencies are plotted vs. time in Figure 4 . These light curves

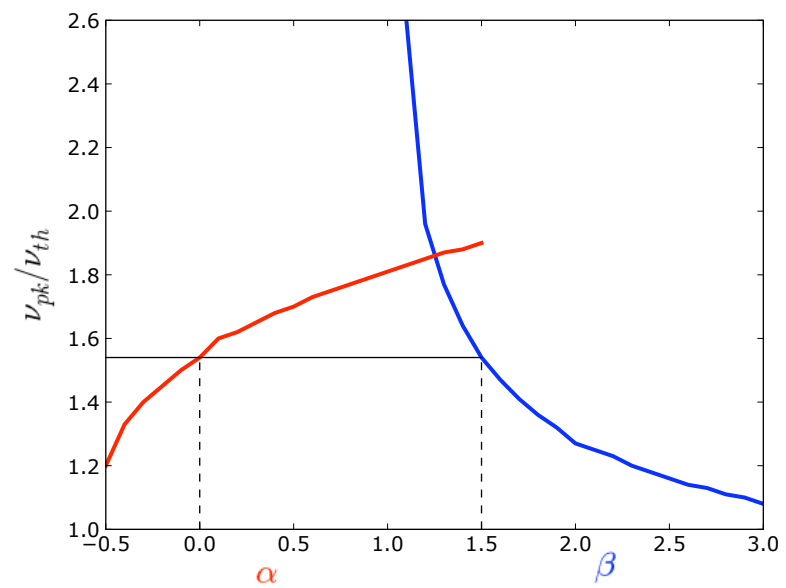

FIG. 3.- Modification of the location of the $\nu F(\nu)$ peak of a Planck spectrum due to the addition of a Comptonized high-frequency tail (blue) or lowfrequency tail (red). Indices in the $\mathrm{x}$-axis are spectral indices $\left(F(\nu) \propto \nu^{-\alpha}\right.$; $\left.F(\nu) \propto \nu^{-\beta}\right)$.

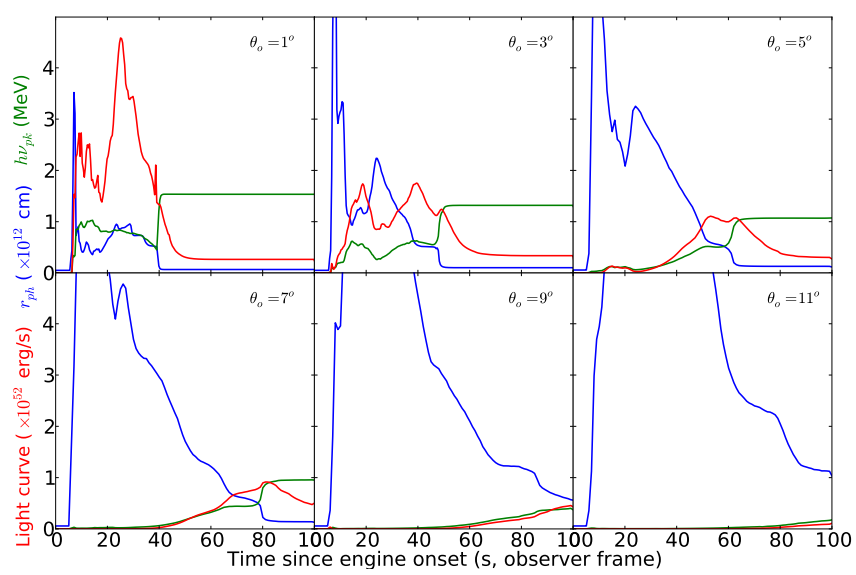

FIG. 4.- Sample results of our post processing for simulation \#1 (see Table 1). Each panel shows a different viewer. Different colors show the bolometric light curve (red), the radius of the photosphere (blue) and the peak frequency (green).

are quite representative also of the other simulations. As a matter of fact, the effects of the viewing angle are much more pronounced than those of a different progenitor, engine duration, or jet properties. As we move away from the jet symmetry axis, all bursts grow weaker, start at a later time, are released at a larger photospheric radius, and are characterized by a smaller photon frequency.

\section{CORRELATIONS}

Figure 5 shows the observed Amati correlation (Amati et al. 2002; 2009) compared to the one found from our synthetic light curves. The change in the location of the radius at which the spectrum is formed has great influence on the synthetic light curves. The typical photon frequency is increased while the total energy is almost unchanged. As a consequence, synthetic bursts agree quantitatively with the Amati correlation in both the slope and the normalization. The agreement results from the combination of two effects. First, the shearing and shocking effects of the stellar material on the outflowing plasma cause a polar stratification of the jet. Different observers see different bursts that, when placed on the Amati diagram, form a stripe along the correlation. In addition, the 


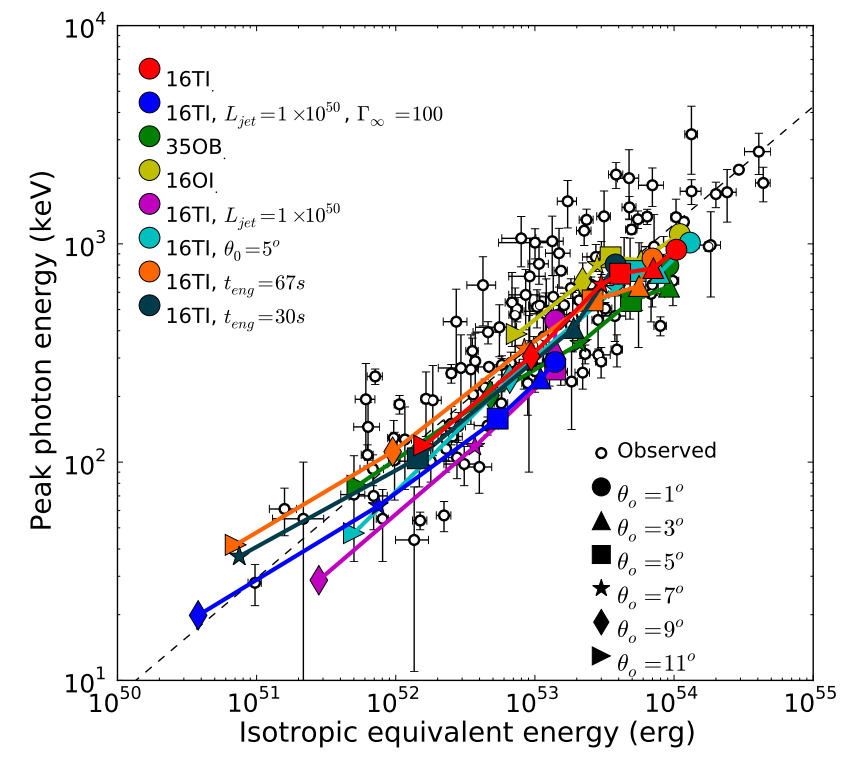

FIG. 5.- Overlay of the simulation results on the observed spectral and energetic properties of gamma-ray bursts. The black symbols with error bars show the observed Amati correlation, i.e., the correlation between the isotropic-equivalent energies of the bursts and the peak photon energies of their $\nu F(\nu)$ spectra (Amati et al. 2002; 2009). The colored lines and symbols show the results of our simulations. Different colors refer to different sets of progenitor and jet properties, as described in the upper left legend. The legend states explicitly the parameters of the simulation that differ from our fiducial progenitor/jet pair (see text and Table 11. Different symbols refer to different orientations of the observer with respect to the jet axis, as described in the lower right legend.

pressure exerted by the stellar material on the jet walls collimates the outflow in such a way as to produce large-scale jets that are insensitive to their initial conditions. We also find that the most important parameter to determine the location of a burst on the Amati diagram is the radius of its stellar progenitor: the slightly bigger 16OI progenitor produces higher peak frequencies at a given energy than the more compact 16TI and 350M progenitors.

The agreement between the synthetic bursts and the observed correlation is quite remarkable since the photospheric model has only a few free parameters: the progenitor star structure, the outflow luminosity and its asymptotic Lorentz factor, and the opening angle of the injected jet. We tried different values for each of these parameters and we always found complete agreement between the simulated and observed bursts. We also tried bursts from engines with different durations. As long as the jet reaches the star surface still relativistic, the computed bursts lie on the Amati correlation. We finally note that our simulations do not cover extreme bursts with very high luminosity and Lorentz factors but are rather targeted to reproduce "average" events. For that reason, none of our simulations reproduces the data in the upper right corner of the figure.

Figure 6 shows a comparison between our results and the observed correlation between the isotropic energy and the Lorentz factor of the outflow (Liang et al. 2010; Ghirlanda et al. 2012a). The observational correlation has significant scatter, possibly due to the difficulty of precisely measuring the Lorentz factor of the outflow. As an example of such difficulty we show the measurements under the assumption of a wind environment with dark empty symbols and the measurements under the assumption of uniform environment with a

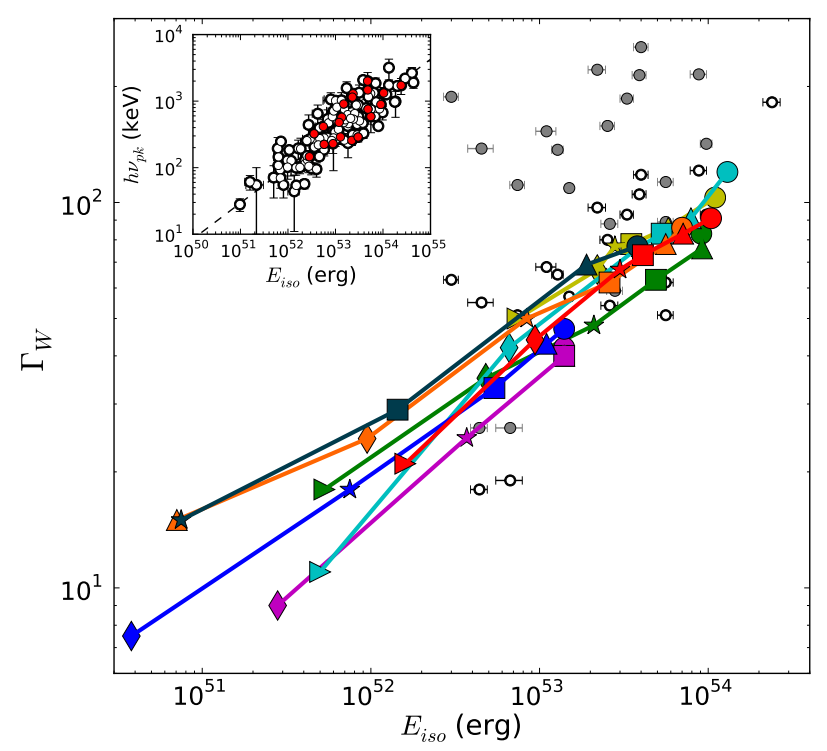

FIG. 6.- Comparison between observed and simulated outflow Lorentz factor versus the burst energy. The black symbols with error bars show the measured Lorentz factor versus the isotropic equivalent energy, assuming that the external shock propagates into a wind environment. The colored lines and symbols show the result of our simulations, quantitatively reproducing the observed correlation. Colors and symbols are the same as in Figure 5 The Lorentz factors from the simulation are computed at the photosphere, since after the photons are released there is no internal pressure left to produce further acceleration of the ejecta. The inset shows the Amati correlation highlighting in red the bursts for which the Lorentz factor could be measured. Finally, smaller gray symbols show the Lorentz factor derived from observations under the assumption of a uniform external medium.

solid gray symbol (from Ghirlanda et al. 2012). The two results are indeed quite different. The Lorentz factor of the synthetic curves is computed as the average of the Lorentz factor of the material at the photospheric radius weighted with the local emissivity. Beyond the photosphere the flow is not accelerated since the radiation decouples and the outflow coasts until the external shock radius is reached, when the observational Lorentz factor is measured. Our simulations reproduce the correlation for a wind environment both in slope and normalization, but they have a smaller scatter. Simulated jets have, however, lower Lorentz factor than the measured values for a uniform environment. Our results extend to lower Lorentz factors with respect to the measurements because it has not been possible to measure the Lorentz factor of weak GRBs, so far (see the inset in Figure 6). The agreement of the simulations with the observations is entirely due to the jet-star interaction, since the simulations adopt a constant $\Gamma_{\infty}=400$ input except for one case, for which $\Gamma_{\infty}=100$ (the blue line and symbols). After the interaction with the stellar material has taken place, the Lorentz factor of the outflow at the photosphere is strongly dependent on the polar angle, in such a way that the observed correlation is reproduced.

Finally, we can use our simulated light curves to compute the radiative efficiency of the synthetic GRB prompt emission. We define the radiation efficiency as the ratio of the energy released as radiation at the photosphere over the total kinetic energy of the flow. We find (see Figure 7) that the simulations predict a broad correlation between peak frequency and radiative efficiency and, transitively, between the total energy radiated and the efficiency.

Measuring the local radiative efficiency from observations 


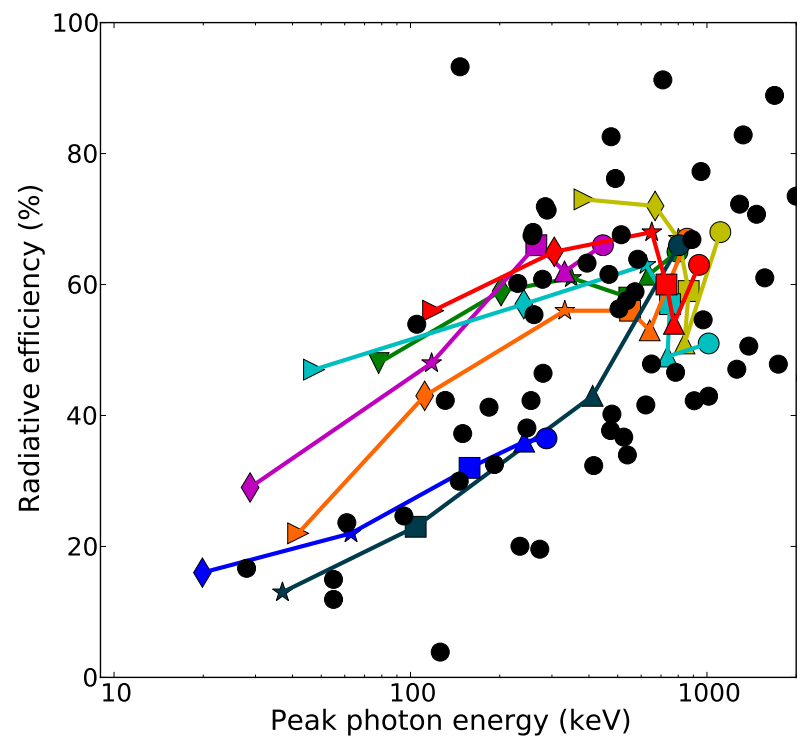

FIG. 7.- Local radiative efficiency of the photospheric emission, defined as the ratio of the energy released in photons to the total energy of the outflow. A tendency of bursts with lower peak frequency (and therefore with lower isotropic-equivalent energy) to have lower efficiency is observed in the simulations. Solid black symbols show local radiative efficiencies estimated by comparing the prompt and early afterglow emission of observed longduration GRBs.

is not an easy task (see, e.g., Zhang et al. 2007), since the measurement must be performed while the outflow is still relativistic in order to avoid contributions from the ejecta moving at large angles with respect to the line of sight. For this reason, radio calorimetry cannot be used. The best proxy to the local radiative efficiency can be obtained, under some assumptions, by comparing the brightness of the prompt emission with that of the early X-ray afterglow. Within the framework of an external shock dominated afterglow, the X-ray emission at early times is produced by electrons in the cooling regime and is fairly insensitive to the density of the ambient medium. Adopting the analytic approximations of Panaitescu \& Kumar (2000) for a wind environment with electrons in slow cooling, we can derive the kinetic energy left in the fireball after the prompt emission as:

$$
\begin{aligned}
E_{k} & =10^{53}\left(\frac{17}{72}\right)^{\frac{p}{p+2}} 10^{\frac{4}{2+p}(0.8 p-59.63)} L_{\nu}^{\frac{p}{2+p}}\left(\frac{\epsilon_{e}}{0.1}\right)^{4 \frac{1-p}{2+p}} \times \\
& \times\left(\frac{\epsilon_{B}}{0.01}\right)^{\frac{2-p}{2+p}}\left(\frac{\nu}{10^{14.6}}\right)^{\frac{2 p}{2+p}}(1+z)^{-\frac{2 p}{2+p}} T_{d}^{\frac{3 p-2}{2+p}}
\end{aligned}
$$

where $p$ is the slope of the non-thermal distribution of electrons, $L_{\nu}$ is the luminosity density at a frequency $\nu$ above the cooling frequency, $\epsilon_{e}$ and $\epsilon_{B}$ are the shock equipartition parameters (i.e., the fraction of the downstream internal energy used to accelerate relativistic electrons and to generate or amplify the magnetic field, respectively), and $T_{d}$ is the time in days after the GRB trigger. Note that Eq. 3 depends very weakly on $\epsilon_{B}$ for a reasonable value of the electron acceleration slope $p \approx 2$ and only the electron equipartition parameter plays a dominant role in the resulting calculation of the kinetic energy (Kumar \& Piran 2000).

In Figure 7 we show the observational efficiencies from a sample of GRBs with X-ray afterglows measurements with solid black symbols. We compute the $E_{X \text {,iso }}$ released in the time interval $(200,250) \mathrm{s}$, rest-frame using the best-fitting light-curves profiles obtained by Margutti et al. (2013) in the common rest-frame energy band $0.3-30 \mathrm{keV}$. Note that this estimate does not include the contribution from X-ray flares. Only GRBs with early afterglow re-pointing have been included and no extrapolation of the observed temporal behavior has been performed. The resulting efficiencies depend on the assumed $\epsilon_{e}$ value and therefore the agreement observed in Figure 7 between the data and observations should be seen as a consistency check rather than a proof. We adopted fairly standerd values in the calculations $\left(\epsilon_{e}=0.1, \epsilon_{B}=0.01\right.$, $p=2.3$ ), but it has to be acknowledged that the quantitative agreement would be lost for a smaller or bigger value of $\epsilon_{e}$ (a qualitative good agreement is obtained for $0.05 \leq \epsilon_{e} \leq 0.2$ ). It is worth mentioning, though, that the general trend of higher efficiency for bursts with larger peak frequency is robust and does not depend on the choice of $\epsilon_{e}$. Finally it should be stressed that at the considered time $(200-250) \mathrm{s}$ there might still be some contribution to the X-ray light curve form the prompt emission. We have also tried to use data from an earlier $(100<t<150 \mathrm{~s})$ and a later $(500<t<750 \mathrm{~s})$ interval, obtaining analogous results.

\section{DISCUSSION AND CONCLUSIONS}

We presented the results of a set of hydrodynamic numerical simulations of the evolution of long-duration GRB jets and their progenitor stars. Thanks to the extended domain of the simulations we were able to derive the location at which the spectral peak of the advected radiation is set and use that information to compute light curves from different progenitors and as seen by observers at different off-axis angles with respect to the stars' rotation axis. We find that within the assumptions made in this study, GRBs produced by photospheric emission reproduce some observational correlations such as the Amati correlation and the energy-Lorentz factor correlation. The ability to account for such correlations, where other radiation mechanisms have failed, suggests that the bulk of the GRB prompt emission is released at the photosphere. Our results also suggest that the primary driver of the difference between very energetic events and weaker ones is the viewing angle, while the scatter in the Amati correlation is due to different jet/progenitor configurations.

Our simulation and the radiation code applied in postprocessing do not follow the full radiation transfer through the outflow and therefore the radiation properties were computed assuming that the spectrum of the entrained radiation is well described at all times by a Planck function in thermal equilibrium with the leptons. In a non-magnetized relativistic jet photon production mechanisms are severely suppressed and the outflow becomes scattering-dominated well before the photospheric radius (Beloborodov 2013; Vurm et al. 2013). Under such conditions, especially in the presence of dissipation, the radiation spectrum would be better approximated by a Wien function with a harder $F(\nu) \propto \nu^{3}$ low-energy tail. We note that, as long as the radiation and leptons are in thermal equilibrium, the peak frequencies of the photons in a Planck and Wien spectrum are equal to within a small factor $\nu_{\text {peak,Planck }} / \nu_{\text {peak,Wien }}=1.07$. Our results are therefore only marginally affected by the choice of a Planck spectrum versus a Wien one. We also note that a detailed discussion of the spectral shape of the photospheric emission is beyond the scope of this paper and we refer the reader to the extensive literature on the formation of non-thermal spectra when subphotospheric dissipation and/or magnetic fields are present 
(Giannios 2006; Pe'er et al. 2006; Giannios \& Spruit 2007; Beloborodov 2010; Lazzati \& Begelman 2010; Vurm et al. 2011).

Another result of our simulations is a strong correlation between the average Lorentz factor of the ejecta that contribute to the light curve and the total radiated energy in the direction to the observer. Such a correlation has been observed (Liang et al. 2010; Ghirlanda et al. 2012) and the results of our simulations are in good agreement with observations as long as a wind environment is assumed in the calculation of the observational Lorentz factor. This is another important piece of the puzzle, since the "standard" synchrotron internal shock model predicts the opposite correlation (Ghisellini et al. 2010). Alternatively, the correlation might be related to the entrainment of baryons from a neutrino-driven wind (Lei et al. 2012). Finally, we found in our simulations that the radiative efficiency of brighter bursts is higher than that of weaker bursts. By comparing the early X-ray afterglow flux to the prompt emission energy of a sample of well-observed bursts, we were able to show that the correlation exists in the observations (see also Bernardini et al. 2012). However, a detailed comparison requires the assumption of a value for the electron equipartition parameter $\epsilon_{e}$, making the quantitative comparison with the simulations parameter-dependent. We show that adopting the fiducial value $\epsilon_{e}=0.1$, we can obtain good agreement between the simulation results and the observed values.
As a final note it should be remembered that the correlations that we discuss here are not exhaustive of all correlations discovered in GRB catalogs. In particular, there is speculation that the more physical correlation is between the peak frequency and the luminosity, rather than the energy release integrated over time (Yonetoku et al. 2004). In addition, an analogous correlation with luminosity exists within individual bursts, where the instantaneous peak frequency correlates with the instantaneous luminosity ( $\mathrm{Lu}$ et al. 2012). Since our simulations assume, for lack of a better model, an engine with constant luminosity, we cannot explore any correlation that depends on the variability injected by the central engine.

We thank S.E. Woosley and A. Heger for making their preSN models available, Lorenzo Amati for sharing his up-todate data on the Amati correlation before publication, and Gabriele Ghisellini and Dimitrios Giannios for valuable discussions. The software used in this work was in part developed by the DOE-supported ASC/Alliance Center for Astrophysical Thermonuclear Flashes at the University of Chicago. This work was supported in part by the Fermi GI program grants NNX10AP55G and NNX12AO74G (DL). BJM is supported by an NSF Astronomy and Astrophysics Postdoctoral Fellowship under award AST-1102796.

\section{REFERENCES}

Amati, L. et al. 2002, A\&A, 390, 81

Amati, L. 2006, MNRAS, 372, 233

Amati, L., Frontera, F., Guidorzi, C. 2009, A\&A, 508, 173

Band, D. et al. 1993, ApJ, 13, 281

Beloborodov, A. M. 2010, MNRAS, 407, 1033

Beloborodov, A. M. 2013, ApJ in press (arXiv:1207.2707

Bernardini, M. G., Margutti, R., Zaninoni, E., Chincarini, G. 2012, MNRAS, 425, 1199

Fan, Y.-Z., Wei, D.-M., Zhang, F.-W., Zhang, B.-B. 2012, ApJ, 755, L6

Fryxell, B. et al. 2000, ApJS, 131, 273

Ghirlanda, G., Nava, L., Ghisellini, G., Firmani, C., Cabrera, J. I. 2008, MNRAS, 387,319

Ghirlanda, G. et al. 2012a, MNRAS, 422, 2553

Ghirlanda, G., Nava, L., Ghisellini, G., Celotti, A., Burlon, D., Covino, S., Melandri, A. 2012b, MNRAS, 420, 483

Ghisellini, G., Celotti, A., Lazzati, D. 2000, MNRAS, 313, L1

Giannios, D. 2006, A\&A, 457, 763

Giannios, D., Spruit, H. C. 2007, A\&A, 469, 1

Giannios, D. 2012, MNRAS, 422, 3092

Kaneko, Y., Preece, R., D., Briggs, M., S., Paciesas, W., S., Meegan, C., A., Band, D., L. 2006, ApJS, 166, 298

Kocevski, D. 2012, ApJ, 747, 146

Kumar, P., Piran, T. 2000, ApJ, 535, 152

Lazzati, D., Morsony, B. J., Begelman, M. C. 2009, ApJ, 700, L47

Lazzati, D., Begelman, M. C. 2010, ApJ, 725, 1137

Lazzati, D., Morsony, B. J., Begelman, M. C. 2011, ApJ, 732, 34

Lei, W.-H., Zhang, B. Liang, E.-W. 2013 arXiv:1209.4427)

Liang, E.-W., Yi, S.-X., Zhang, J.; Lü, H.-J., Zhang, B.-B., Zhang, B. 2012, ApJ, 725, 2209
López-Cámara, D., Morsony, B. J., Begelman, M. C., Lazzati, D. 2013, ApJ submitted (arXiv:1212.0539)

Lu, R.-J. et al. 2012, ApJ, 756, 112

Margutti, R. et al. 2013, MNRAS, 28, 729

Mészáros, P., Rees, M. J. 2000, ApJ, 530, 292

Mizuta, A., Nagataki, S., Aoki, J. 2011, ApJ, 732, 26

Morsony, B. J., Lazzati, D., Begelman, M. C. 2007, ApJ, 665, 569

Nagakura, H., Ito, H., Kiuchi, K., Yamada, S. 2011, ApJ, 731, 80

Nakar, E., Piran, T. 2005, MNRAS, 360, L73

Panaitescu, A., Kumar, P. 2000, ApJ, 543, 66

Pe'er, A., Mészáros, P., Rees, M. J. 2005, ApJ, 635, 476

Pe'er, A., Mészáros, P., Rees, M. J. 2006, ApJ, 642, 995

Piran, T. 1999, Phys. Rep., 314, 575

Rees, M., J., Mészáros, P. 1994, ApJ, 430, L93

Rees, M., J., Mészáros, P. 2005, ApJ, 628, 847

Thompson, C., Mészáros, P., Rees, M. J. 2007, ApJ, 666, 1012

Vurm, I., Beloborodov, A. M., Poutanen, J. 2011, ApJ, 738, 77

Vurm, I., Lyubarsky, Y., Piran, T. 2013, ApJ in press (arXiv:1209.0763)

Woosley, S. E., Heger, A. 2006, ApJ, 637, 914

Yonetoku, D., Murakami, T., Nakamura, T., Yamazaki, R., Inoue, A. K.,

Ioka, K. 2004, ApJ, 609, 935

Zhang, B. et al. 2007, ApJ, 655, 989

Zhang, B., Yan, H. 2011, ApJ, 726, 90

Zhang, B.-B. et al. 2011, ApJ, 730, 141 